\title{
Amadeus
}

International Multidisciplinary Journal IISSN 2525-3281

DOI: 10.14295/aimj.v5i9.149

\section{Treatment of American Tegumentary Leishmaniosis with Fluconazole: Case Report}

Kauane Mayra de Oliveira Xavier ${ }^{1}$,

Rainardo Antônio

Puster $^{2}$,

Hermes Melo

Teixeira Batista ${ }^{3}$,

Jorge Luiz

Nobre Rodrigues ${ }^{4}$.

\begin{abstract}
Leishmaniasis are diseases caused by protozoan parasites of the Leishmania genus, seen as a major public and social health problem, they represent a complex of diseases with different clinical presentations. Pentavalent antimonial drugs are the first choice in their treatment, however, this medications have disadvantages, such as the application and risks attributed to the toxicity. In this context, therapeutic options that present a suitable intake and satisfactory results are studied. The authors present a clinical case of American cutaneous leishmaniasis in a male patient, 89 years old, with cutaneous lesions on the nasal dorsum and on the left infraorbital margin, after the diagnosis, therapy with oral fluconazole was started, thus improving the disease. Oral triazole antifungals are a controversial topic in cutaneous leishmaniasis, even so, the use of oral fluconazole can be considered in patients with skin lesions, especially in patients susceptible to the greater possibility of complications due to side effects of pentavalent antimonial drugs.
\end{abstract}

Keywords: American Cutaneous Leishmaniasis. Fluconazole. Public health.

\footnotetext{
${ }^{1}$ Medical Resident in the Medical Clinic program at the Federal University of Ceará - Walter Cantídio University Hospital (HUWC). E-mail: kauanexavier@yahoo.com.br;

${ }^{2}$ Tutor. Preceptor and vice-supervisor of the Medical Clinic Program at the University Hospital Walter Cantídio of UFC. E-mail: rainardopuster@gmail.com;

${ }^{3} \mathrm{PhD}$ in Health Sciences by FMABC. Professor at the Faculty of Medicine of Araripina FAP. hermes2710batista@gmail.com;

${ }^{4} \mathrm{PhD}$ in Infectious and Parasitic Diseases at Escola Paulista de Medicina. Preceptor of the Infectious Diseases Program at the Walter Cantídio UFC University Hospital. E-mail: jorge1207@ gmail.com.
} 


\section{Tratamento da Leishmaniose Tegumentar Americana com Fluconazol: Relato de Caso}

\begin{abstract}
Resumo: As leishmanioses são doenças causadas por parasitas protozoários do gênero Leishmania, encaradas como um grande problema de saúde pública e social, elas representam um complexo de doenças com diferentes apresentações clínicas. Os fármacos antimoniais pentavalentes constituem a primeira escolha no seu tratamento, porém, tais medicações apresentam desvantagens, como a forma de aplicação e riscos atribuídos à sua toxicidade. Neste contexto, são estudadas opções de terapêutica que apresentem resultados satisfatórios e de posologia conveniente. Os autores apresentam um caso clínico de leishmaniose tegumentar americana em um paciente do sexo masculino, 89 anos, com lesões cutâneas em dorso nasal e em margem infraorbitária esquerda, após o diagnóstico, foi iniciada terapêutica com fluconazol oral, ocorrendo assim a melhora da doença. Os antifúngicos triazólicos orais são tema controverso na leishmaniose tegumentar, ainda assim, o uso de fluconazol oral pode ser considerado em pacientes com lesões cutâneas, principalmente em pacientes suscetíveis a maior possibilidade de complicações por efeitos colaterais de fármacos antimoniais pentavalentes.
\end{abstract}

Palavras-Chave: Leishmaniose Tegumentar Americana. Fluconazol. Saúde Pública.

\section{Introdução}

As leishmanioses são reconhecidas como um grande problema de saúde pública, representam um complexo de doenças com apresentação variada. Segundo a Organização Mundial da Saúde, 350 milhões de pessoas em todo o mundo estão expostas ao risco de contrair alguma forma de doença ${ }^{1}$.

Diferentes síndromes clínicas são incluídas sob a infecção por leishmanias, e alguns fatores interferem nessas apresentações, como a resposta imunológica do hospedeiro e elementos inerentes ao protozoário. Em relação ao espectro tegumentar, as principais apresentações são: leishmaniose cutânea, mucosa, cutânea difusa e disseminada ${ }^{2,3}$. 
A partir dos anos 40, drogas de administração parenteral, representam alicerce no tratamento para leishmaniose. Os fármacos antimoniais pentavalentes constituem a primeira escolha para ela, entretanto, tais drogas apresentam desvantagens e riscos, os quais são: a forma da aplicação, a duração do tratamento e sua toxicidade (por exemplo, fadiga, anormalidades eletrocardiográficas, alterações hepáticas, renais, risco de pancreatite química). Em casos resistentes, a anfotericina B é uma alternativa geralmente eficaz, porém, de segunda linha pelo risco frequente de causar efeitos tóxicos irreversíveis ${ }^{4,5}$. Neste contexto, são estudadas opções de terapêutica com resultados satisfatórios e de posologia conveniente, na doença primária, na infecção persistente, recidivante e no tratamento de pacientes em maior risco de efeitos adversos como idosos, gestantes e diabéticos ${ }^{6}$.

Os antifúngicos triazólicos orais são tema controverso no tratamento da leishmaniose tegumentar americana, mesmo assim, seu uso pode ser considerado em lesões cutâneas complexas que têm potencial para evoluir para doença mucocutânea ${ }^{7}$.

\section{Relato de Caso}

Paciente do sexo masculino, 89 anos, pardo, aposentado como agricultor, natural e procedente de Maranguape, compareceu ao serviço de dermatologia do Hospital Universitário Walter Cantídio, devido ao surgimento de lesões assintomáticas em região em dorso nasal e infra-orbitária esquerda, com 02 anos de evolução, o paciente permaneceu durante esse período afebril, sem apresentar adenopatias ou visceromegalias, no início do quadro realizou tratamento com solução de ácido salicílico e ácido lático sem melhora clínica.

Ao exame físico, apresentava placas infiltrativas com eritema, áreas de erosão e descamação, de consistência endurecida em região infra-orbitária esquerda. Notava-se ainda lesão infiltrativa em dorso nasal (Figura 1). 
Figura 1. Lesões em placas, eritematosas e infiltrativas em região infraorbitária esquerda e dorso nasal

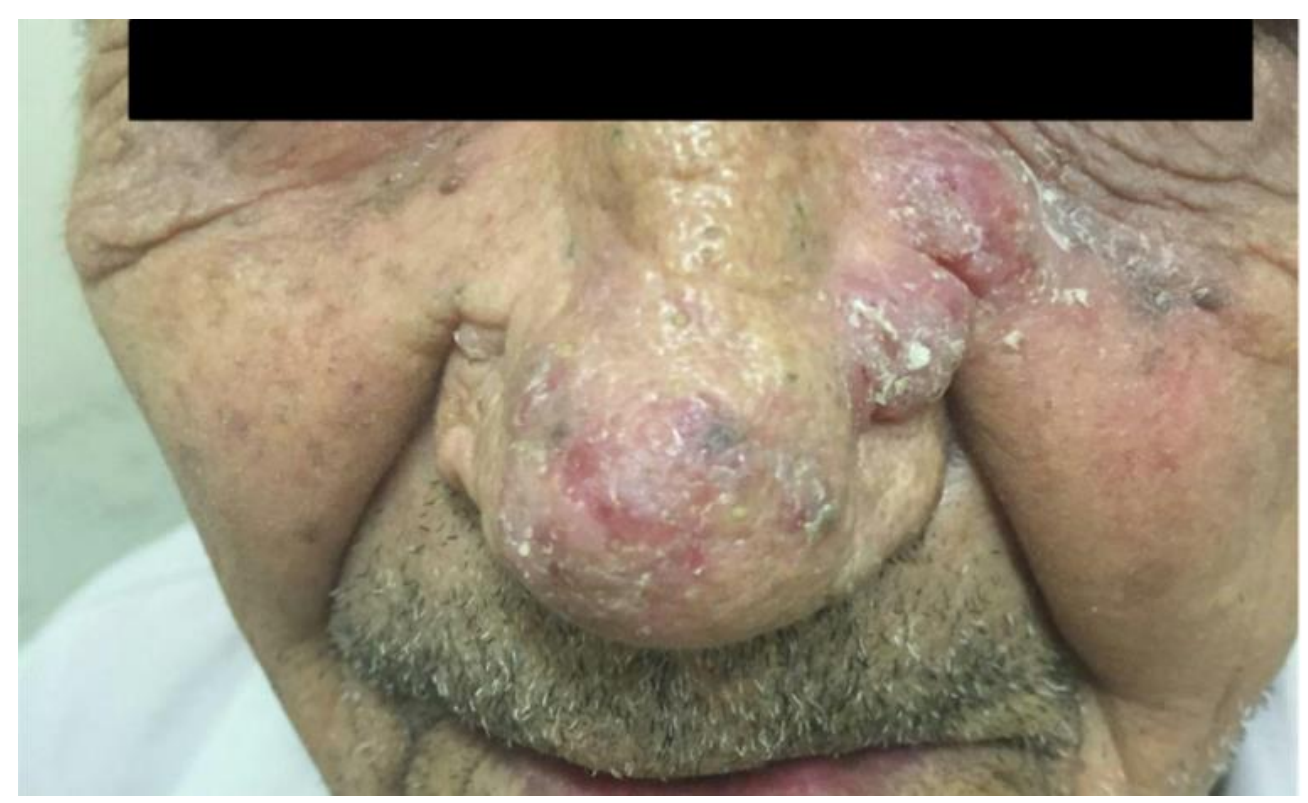

Fonte: Dados da pesquisa. Arquivo dos autores, 2020.

A partir de então, foram feitas as seguintes hipóteses diagnósticas: doença linfoproliferativa, tuberculose cutânea, leishmaniose tegumentar americana, sarcoidose e o granuloma facial.

Após biopsia de lesão em dorso nasal por punch, a revisão de lâmina histopatológica evidenciou infiltrado linfoplasmocitário com resultado negativo para micobactérias e fungos, foi então submetida à imunohistoquímica que revelou processo infeccioso por Leishmanias.

Diagnosticada a doença, foi iniciado tratamento oral com fluconazol 450mg por dia para leishmaniose tegumentar americana por 06 semanas e acompanhamento com realização de exames como função hepática, notada a resposta durante as primeiras semanas, foi então mantido tratamento por mais 04 semanas (Figura 2). Nenhum efeito colateral foi relatado pelo paciente ou pelo médico assistente. 
Figura 2. Evolução das lesões após 10 semanas de tratamento

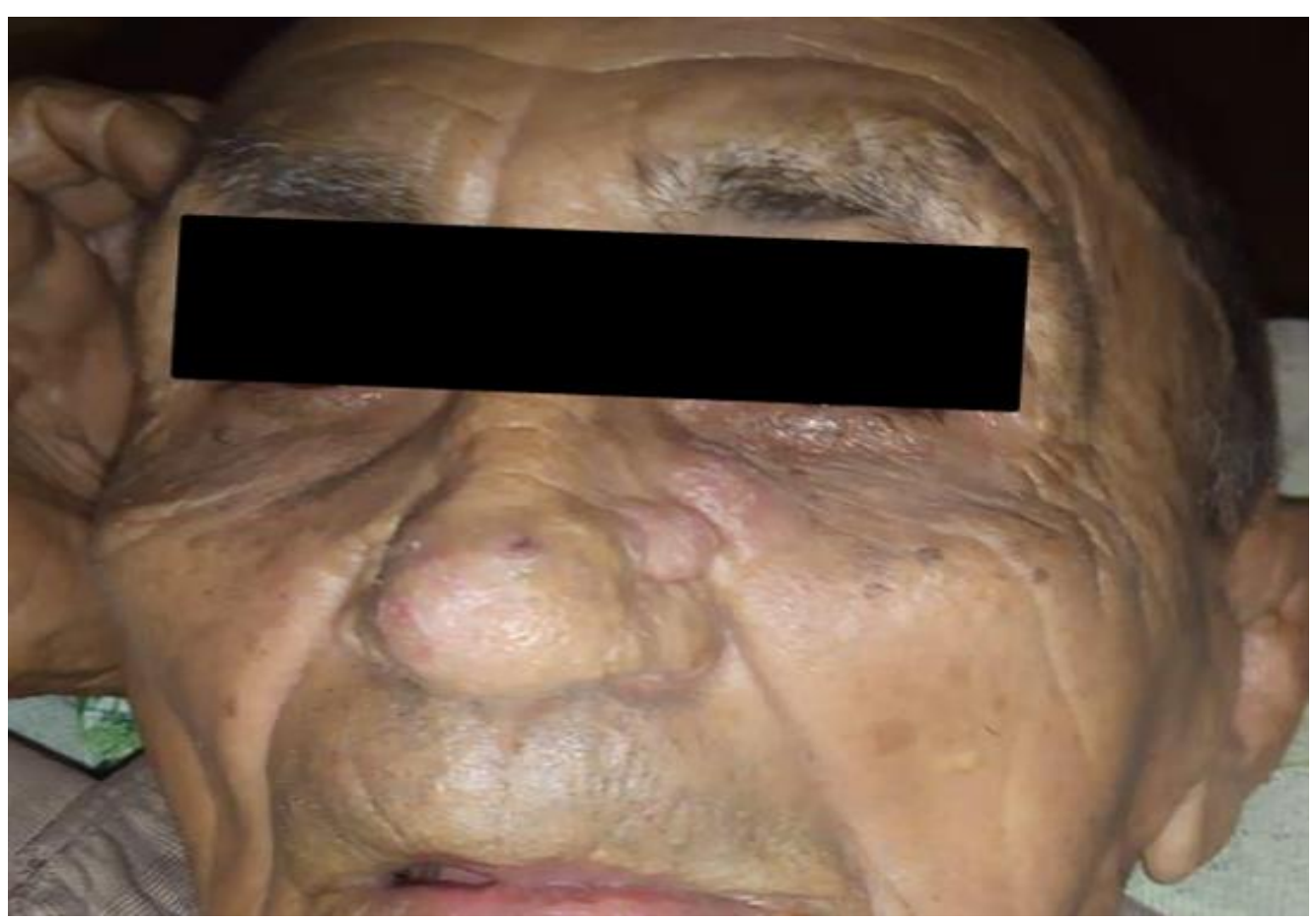

Fonte: Dados da pesquisa. Arquivo dos autores, 2020.

\section{Discussão}

Do ponto de vista epidemiológico, no estado do Ceará, um levantamento realizado no período de 2007 a 2016, detectou a semelhança de características com o restante do país, em que a leishmaniose de forma cutânea foi a mais frequente, existindo também correlação com a maioria do número de casos oriundos de regiões endêmicas com transmissão autóctone ${ }^{8}$.

A leishmaniose tegumentar americana é uma doença decorrente da infecção por parasitas nos macrófagos do sistema fagocitário mononuclear. A maioria dos pacientes apresenta lesões que variam em tamanho de 0,5 a $3 \mathrm{~cm}$ de diâmetro, geralmente em partes expostas da face, braços ou pernas. Os pacientes podem ter várias lesões; algumas crescem, mas não ulceram, formando nódulos 2,3 .

Neste artigo, relata-se um caso de leishmaniose tegumentar americana em um paciente que apresentava lesão granulomatosa na face. A lesão permitiria diferentes diagnósticos diferenciais, o diagnóstico foi realizado após análise imuno-histoquímica. 
O carater crônico da leishmaniose tegumentar também pode se assemelhar clinicamente a tuberculose cutânea e a hanseniase tuberculoide ${ }^{4}$. Por causa da inflamação crônica de uma infecção por Leishmania, um câncer de pele primário pode se desenvolver no local de uma antiga cicatriz ${ }^{9}$.

Um dos grandes desafios no diagnóstico é a realização de cultura para a identificação da espécie do parasita. Apesar de uma especifidade de aproximadamente 100\%, a taxa de sensibilidade da cultura é de apenas $40 \%{ }^{10}$. Encontram-se aspectos relevantes de achados imunohistoquímicos na identificação da leishmaniose tegumentar. Na infecção pela leishmaniose as primeiras células afetadas pelo protozoário são as células dendríticas, células de Langerhans, dendrócitos dérmicos e macrófagos ${ }^{11}$. O diagnóstico de certeza é feito pelo encontro do protozoário, a comprovação clínico-epidemiológica da infecção deve ser complementada pela pesquisa direta. A identificação da espécie de Leishmania mostra-se um dado importante para a epidemiologia e para o tratamento ${ }^{1}$.

Existe uma ligação entre a apresentação clínica e os achados histológicos da doença. Um grande número de amastigotas e de histiócitos sem outras células inflamatórias sugerem uma reação imunitária fraca, enquanto uma quantidade escassa de amastigotas é geralmente relacionada ao aparecimento de granulomas ou necrose ${ }^{2}$.

Um dos pilares da grande problemática no tratamento com triazólicos é que ainda demonstram-se necessários estudos respaldados. A variação da resposta ocorre dependente da espécie do protozoário, da escolha da medicação, do tamanho da lesão, das comorbidades atreladas ao paciente, do espectro clínico apresentado, do tempo de tratamento a ser realizado, a alternativa na recaída e o uso de mais de uma posologia ${ }^{12}$.

O uso dos antimoniais pentavalentes, tratamento de primeira escolha, deve ser evitado em pacientes acima dos 50 anos de idade, portadores de cardiopatias, nefropatias, hepatopatias e deve-se poupar o uso conjunto de medicamentos que provocam alterações eletrocardiográficas ${ }^{4}$.

Em relação aos antifúngicos azólicos, alguns de apresentação oral podem ser empregados no tratamento da leishmaniose tegumentar. Diferentes moléculas podem ser testadas: fluconazol (200 mg/dia por 6 semanas); itraconazol (100-400 mg/dia por 6-8 semanas); e cetoconazol (200-600 mg/dia durante 28 dias $)^{10,13}$. Drogas que atuam sobre a biossíntese da membrana celular do protozoário. ${ }^{14}$ Seu uso é muitas vezes limitado pela toxicidade hepática que podem ocasionar, além de sua eficiência variar de resultados entre os estudos ${ }^{10,13}$.

No caso relatado, por conta da idade avançada do paciente e risco de efeitos adversos atrelados ao tratamento convencional, foi estabelecido iniciar tratamento alternativo com 
fluconazol, droga de maior comodidade posológica e mais segura no manejo em acompanhamento ambulatorial.

Em um ensaio clínico duplo cego, randomizado e controlado por placebo realizado em 2002, na Arábia Saudita, sobre a eficácia do fluconazol na leishmaniose cutânea causada por Leishmania major, com a dose de $200 \mathrm{mg}$ ao dia por 6 semanas, observou-se a remissão completa no seguimento de 3 meses em 63 dos 80 pacientes no grupo tratado com fluconazol (79\%) e em 22 dos 65 pacientes no grupo placebo (34\%). Além disso, o tempo de cicatrização foi menor no grupo que utilizou o fármaco (8,5 semanas) em comparação com placebo $^{15}$.

Em um estudo realizado com 28 pessoas, em uma regiao endêmica para Leishmania braziliensis, o tratamento com fluconazol na dose de $8 \mathrm{mg} / \mathrm{kg}$ por dia mostrou-se uma boa alternativa do ponto de vista econômico, além de bem tolerado, apresentou resposta clínica convicente 6 .

Embora o tratamento tradicionalmente empregado para a maioria dos casos seja realizado com antimoniais pentavalentes, estudos recentes exploram alternativas da terapia que facilitem a administração. $O$ uso do fluconazol oral surge como uma opção atraente ao tratamento da doença.

\section{Referências}

1. Ministério da Saúde. Secretaria de Vigilância em Saúde. Departamento de Vigilância das Doenças Transmissíveis. Manual de vigilância da leishmaniose tegumentar. [Internet]. Brasília; 2017 [acesso em: 06 nov. 2018]. Disponível em http://bvsms.saude.gov.br/bvs/publicacoes/manual_vigilancia_leishmaniose_tegumentar.pdf

2. Díaz SJ, Barrientos SS, Morell MS. Leishmaniasis cutánea. FMC - Formación Médica Continuada en Atención Primaria. 2012;19(3):117-28.

3. Guimarães LH, Machado PR, Lessa LH, Lessa M, D’Oliveira AJ, Carvalho EM. Aspectos Clínicos da Leishmaniose Tegumentar. Gazeta Médica Bahia, 2005; 75(1):66-74.

4. Handler M, Patel P, Kapila R, Al-Qubati Y, Schwartz R. Cutaneous and mucocutaneous leishmaniasis. Journal of the American Academy of Dermatology. 2015;73(6):911-26.

5. Herwaldt B. Leishmaniasis. The Lancet. 1999;354(9185):1191-99.

6. Sousa A, Frutuoso M, Moraes E, Pearson R, Pompeu M. High-Dose Oral Fluconazole Therapy Effective for Cutaneous Leishmaniasis Due to Leishmania (Vianna) braziliensis. Clinical Infectious Diseases. 2011;53(7):693-95. 
7. Bailey M, Lockwood D. Cutaneous leishmaniasis. Clinics in Dermatology. 2007;25(2):20311.

8. Cunha JCL, Cardoso ARP, Feijão LX, Crisóstomo BS, Oliveira RP. Aspectos clínicos e epidemiológicos da leishmaniose tegumentar americana no estado do Ceará, Brasil, no período de 2007 a 2016. Cadernos ESP, Ceará. 2017; 11 (2):10-17.

9. Kopterides P, Mourtzoukou E, Skopelitis E, Tsavaris N, Falagas M. Aspects of the association between leishmaniasis and malignant disorders. Transactions of the Royal Society of Tropical Medicine and Hygiene. 2007;101(12):1181-89.

10. Masmoudi A, Hariz W, Marrekchi S, Amouri M, Turki H. Old World cutaneous leishmaniasis: diagnosis and treatment. Journal of Dermatological Case Reports. 2013; 7(2): $31-41$.

11. Duarte M, Rochael M. Perfil histopatológico e imuno-histoquímico da leishmaniose tegumentar americana com ênfase nos dendrócitos dérmicos FXIIIa+. Anais Brasileiros de Dermatologia. 2006;81(6):541-48.

12. Reithinger R, Dujardin J, Louzir H, Pirmez C, Alexander B, Brooker S. Cutaneous leishmaniasis. The Lancet Infectious Diseases. 2007;7(9):581-96.

13. Minodier P, Parola P. Cutaneous leishmaniasis treatment. Travel Medicine and Infectious Disease. 2007;5(3):150-58.

14. Beach D, Goad L, Holz G. Effects of antimycotic azoles on growth and sterol biosynthesis of Leishmania promastigotes. Molecular and Biochemical Parasitology. 1988;31(2):149-62.

15. Alrajhi A, Ibrahim E, De Vol E, Khairat M, Faris R, Maguire J. Fluconazole for the Treatment of Cutaneous Leishmaniasis Caused by Leishmania major. New England Journal of Medicine. 2002;346(12):891-95.

\section{How to cite this article (APA format):}

Xavier, Kauane Mayra de Oliveira; Puster, Rainardo Antônio; Batista, Hermes Melo Teixeira; Rodrigues, Jorge Luiz Nobre (2020). Treatment of American Tegumentary Leishmaniosis with Fluconazole: Case Report. Am. In. Mult. J., Jul to Dec (9) 5, 294-301.

Received: 11/11/2020;

Accepted: 11/26/2020. 